\title{
Resting Tremor due to Risperidone Long-Acting Injection
}

\author{
Halil Onder
}

A-51-year-old female patient with a medical history of bipolar disorder (for 5 years) and temporal lobe epilepsy (for 10 years) was consulted to our department due to right-sided resting tremor which started 6 months ago and gradually progressed in the interval period. Upon interrogation of medical history, it was learned that the patient had been taking valproic acid $1,000 \mathrm{mg}$ and carbamazepine $400 \mathrm{mg}$ for the last 3 years. However, risperidone long-acting injection (RLAI) was also initiated 8 months ago ( $37.5 \mathrm{mg}$ biweekly) due to manic relapse and psychotic behaviors. Remarkably, the patient and her relatives stated that resting tremor had started nearly 2 months after initiation of RLAI therapy. Of note, no family history of an early Parkinson's disease was taken and there was no kinship between parents. On neurological examination, the patient was fully orientated and cooperative. Motor and sensorial examinations were normal; however, 4- to 6-Hz pill-rolling tremor in the resting right upper limb was recognized. The tremor was less prominent during posture and action. Other extrapyramidal system examinations revealed a slight bradykinesia in the right hand and bradyphrenia. Rigidity and Myerson's sign was absent. Conventional brain MRI was evaluated within normal limits (no a vascular lesion or a significant atrophy of any region). With a provisional diagnosis of drug-induced tardive parkinsonism (in the form of resting tremor), the patient was re-evaluated together with the psychiatry department. Considering that overdose of valproic acid is known to cause tremor, serum valproic acid level was studied two times which revealed valproic acid level at the lower limit of the range $(51 \mu \mathrm{g} / \mathrm{mL}$ and $54 \mu \mathrm{g} / \mathrm{mL}$, respectively; range:50 - 100). Taken together the laboratory findings and clinical course (the tremor was during resting which is incompatible with the valproic acid tremor that is generally significant during posture, clinical course was rapid for PD and association of temporal evolution of the clinic with risperidone therapy), the tremor was associated with risperidone usage. Risperidone was switched to aripiprazole $10 \mathrm{mg}$ therapy and policlinic follow-up visits were planned. On the third week of follow-up, no improvement in tremor (as well as bradykinesia) was detected. However, further followup was planned. Finally, on the third month of follow-up on valproic acid 1,000 mg, carbamazepine 400 and aripiprazole $10 \mathrm{mg}$ therapies, a dramatic improvement was achieved that resting tremor was totally recovered.

Manuscript submitted October 23, 2018, accepted November 12, 2018

Department of Neurology, Yozgat City Hospital, Yozgat, Turkey. Email: halilnder@yahoo.com

doi: https://doi.org/10.14740/jnr505
Neuroleptic-induced parkinsonism (NIP) is known to begin within weeks after starting an antipsychotic medication [1]. NIP is generally treated by cessation of the inducing neuroleptic drugs. However, in patients who need to continue antipsychotic medications, antipsychotic treatment may be switched to atypical antipsychotics which have a lower risk of extrapyramidal side effects [2]. Generally, atypical antipsychotics have substantially a lower risk of developing tardive syndromes in comparison to traditional ones due to their lower propensity for causing antidopaminergic side effects [3]. This feature has been associated with their specific feature of fast dissociation from D2 receptors [4]. However, in some recent reports, it has been called into question [1]. Remarkably, in recent two trials evaluating patients on risperidone therapy, a considerable number of patients were reported to develop extrapyramidal side effects which had led to the initiation of anticholinergic therapy $[5,6]$. Besides, in a randomized, double-blind, placebo-controlled study investigating the follow-up data of patients on maintenance treatment with adjunctive RLAI, the tremor was found to be as a common adverse event in RLAI group according to the placebo group (24.6\% versus $10.2 \%$ ) [7]. Of note, no description regarding the phenomenology of tremor (resting or postural or intention?) was included in this report avoiding to make further comments. On the other hand, in a systematic review of large pediatric patients group including 737 patients treated with risperidone, tardive syndrome was found to develop in only two of the them [8].

In this report, I illustrate the clinical course of an extremely rare patient, diagnosed with neuroleptic induced parkinsonism due to RLAI therapy who was finally fully recovered following switch to aripiprazole treatment on the third month followup. Similarly, Chung et al reported a patient developing tardive dyskinesia associated with RLAI who had recovered after switching aripiprazole treatment [9]. The authors suggested that faster dissociation of aripiprazole than risperidone might be an explanation underlying this significant improvement [9]. Besides, they discussed its (aripiprazole) partial agonistic D2 receptor, 5HT1a receptor activity, and its limited potential to cause up-regulation in either $\mathrm{D} 2$ receptor binding sites or D2 mRNA as possible other mechanisms to explain the resolution of the clinic [9]. To the best of my knowledge, tardive parkinsonism associated with risperidone usage has been reported in only a single previous report which had occurred in a patient with schizophrenia [10]. However, this index case was on follow-up due to bipolar disorder, rest-tremor was the prominent sign which was not in the case by Chen et al [10] and the causative agent was RLAI (not oral risperidone). Hence, I think that the presentation of this case is a unique sample which may 
add substantial perspectives in this area.

Taken together, via the presentation of this case and literature knowledge, I re-emphasize to keep in mind tardive parkinsonism in patients with atypical presentations and history of antipsychotics usage, even the atypical ones such as risperidone. In these patients, switching to other antipsychotic medications should be considered before anticholinergic agents and levodopa trial. I think that the effectiveness of switching to aripiprazole therapy should be particularly investigated in future larger patients group, perhaps in the subgroup of tardive parkinsonism. In addition, I would like to remark that longterm follow-up may be required for the final diagnosis, as the resolution of symptoms may occur within 6 months after the causative agent is suspended [11] which persisted more than 2 months in our case. However, as functional neuroimaging studies lack in this report, we cannot exclude a disposition to develop idiopathic PD which might have manifested earlier on the setting of antipsychotic usage. Nevertheless, a nearly totally resolution of symptoms was achieved after cessation of RLAI refuting this hypothesis. Future larger studies using multimodal neuroimaging techniques are surely warranted which may add substantial contributions regarding the pathophysiology of parkinsonism as well as efficient pathways of atypical neuroleptics.

\section{Conflict of Interest}

None.

\section{Funding}

None.

\section{References}

1. Pringsheim T. Missed diagnosis of tardive dystonia in an adolescent girl treated with risperidone. J Child Adolesc Psychopharmacol. 2011;21(2):191-194.

2. Shin HW, Chung SJ. Drug-induced parkinsonism. J Clin
Neurol. 2012;8(1):15-21.

3. Correll CU, Leucht S, Kane JM. Lower risk for tardive dyskinesia associated with second-generation antipsychotics: a systematic review of 1-year studies. Am J Psychiatry. 2004;161(3):414-425.

4. Seeman P. An update of fast-off dopamine D2 atypical antipsychotics. Am J Psychiatry. 2005;162(10):19841985.

5. Sikich L, Frazier JA, McClellan J, Findling RL, Vitiello B, Ritz L, Ambler D, et al. Double-blind comparison of first- and second-generation antipsychotics in earlyonset schizophrenia and schizo-affective disorder: findings from the treatment of early-onset schizophrenia spectrum disorders (TEOSS) study. Am J Psychiatry. 2008;165(11):1420-1431.

6. Sikich L, Hamer RM, Bashford RA, Sheitman BB, Lieberman JA. A pilot study of risperidone, olanzapine, and haloperidol in psychotic youth: a double-blind, randomized, 8-week trial. Neuropsychopharmacology. 2004;29(1):133-145.

7. Macfadden W, Alphs L, Haskins JT, Turner N, Turkoz I, Bossie C, Kujawa M, et al. A randomized, double-blind, placebo-controlled study of maintenance treatment with adjunctive risperidone long-acting therapy in patients with bipolar I disorder who relapse frequently. Bipolar Disord. 2009;11(8):827-839.

8. Correll CU, Kane JM. One-year incidence rates of tardive dyskinesia in children and adolescents treated with second-generation antipsychotics: a systematic review. J Child Adolesc Psychopharmacol. 2007;17(5):647-656.

9. Chung AK. Atypical presentation of tardive dyskinesia associated with risperidone long-acting injection as maintenance treatment in bipolar affective disorder: a case report. Curr Drug Saf. 2012;7(1):21-23.

10. Chen $\mathrm{CK}, \mathrm{Wu} \mathrm{JH}$. Improvement of risperidone-related tardive parkinsonism with a switch to aripiprazole. Prog Neuropsychopharmacol Biol Psychiatry. 2009;33(7):1279-1280.

11. Bower JH, Maraganore DM, McDonnell SK, Rocca WA. Incidence and distribution of parkinsonism in Olmsted County, Minnesota, 1976-1990. Neurology. 1999;52(6):1214-1220. 